\title{
Robust Event-based Data Scheduling for Resource Constrained Networked Control Systems
}

\author{
Mohammad H. Mamduhi ${ }^{1}$, Domagoj Tolić ${ }^{2}$ and Sandra Hirche ${ }^{1}$
}

\begin{abstract}
This paper modifies a recently proposed eventbased probabilistic medium access for multi-loop Networked Control Systems (NCSs) over a shared communication channel subject to limited capacity and uncertainties and study its robustness. The novel design combines deterministic and probabilistic attributes to efficiently allocate the channel access among the control loops in the presence of network-induced phenomena such as packet dropouts and scheduling with delayed information update. Since the scheduler receives error information from a number of systems simultaneously, this sheer amount of information cannot always be processed in timely manner, which in turn gives rise to delays. Given the local error thresholds, the subsystems with error values lower than their corresponding thresholds are deterministically excluded from the medium access competition in favor of those with larger errors. In case of resource scarcity, the scheduler probabilistically allocates the channel to those that exceed the local thresholds according to an error-dependent priority measure. We show stochastic stability of such NCSs in terms of $f$-ergodicity of the network-induced error, which is modeled as a Markov chain. Numerical results validate our stability results in the presence of packet dropouts and delayed data update.
\end{abstract}

\section{INTRODUCTION}

Control over shared communication resources imposes various challenges, such as capacity limitation, collision, time delays and packet dropouts, that impair the control performance and can even lead to instability. In order to efficiently utilize the limited communication and energy resources, event-triggered control and scheduling designs have been proposed [1]-[4]. The references suggest that it is often more beneficial to transmit the sampled data upon the occurrence of certain events rather than at predefined time instants, especially when large-scale systems are of interest.

While time-triggered protocols usually offer lower complexity as they employ periodic offline access schemes, event-based protocols excel in efficient resource allocation, scalability, and robustness. In the event-based paradigm, events are typically triggered by either deterministic [5], [6], or stochastic policies [7]-[10]. Try-Once-Discard (TOD) is a basic deterministic event-based protocol which awards the medium access to the system with the largest estimation error, and discards the remaining requests [6]. However, TOD is vulnerable to noise and can cope with collisions only when a priority order is predefined. The Maximal Allowable

\footnotetext{
${ }^{1} \mathrm{M}$. H. Mamduhi and S. Hirche are with the Institute for Informationoriented Control, Technische Universität München, Arcisstraße 21, D-80290 München, Germany; \{mh.mamduhi, hirche\}@tum. de

${ }^{2}$ Domagoj Tolić is with the University of Zagreb, Faculty of Electrical Engineering and Computing, Unska 3, 10000 Zagreb, Croatia. His work is supported by the European Community Seventh Framework Programme under grant No. 285939 (ACROSS). domagoj. tolicefer.hr
}

Transfer Interval (MATI) is employed for stability analysis of systems with deterministic medium access schemes [5], [6]. However, MATI is not applicable to stochastic schemes as the intervals between consecutive transmissions usually cannot be upper bounded uniformly. This calls for new approaches regarding stability of stochastic NCSs [7]-[10]. While the scheduling architectures for single-loop NCSs are well addressed ( [8], [9]), further studies for multi-loop NCSs are required ( [7], [10] are notable exceptions).

Robust control and scheduling designs for NCSs over non-ideal communication mediums are also widely explored [11]-[16]. Packet dropouts are modeled either as stochastic [14], or deterministic [12] phenomena. In the latter references, worst-case bounds for the number of consecutive dropouts are derived. Stability of time-invariant systems is studied in [13] for constant delays, while protocols with timevarying delays are discussed in [15]. A scheduling design with uncertain delays is presented in [11]. Robustness of scheduling policies regarding the availability of information is not investigated to the best of the authors' knowledge. Scheduling approaches requiring complete information in every time instant might not be feasible in practice due to the additional traffic imposed by the scheduler to coordinate among different control loops. A desired scheduling approach should be capable of allocating the resource efficiently provided with partial information from local entities.

In this paper, we extend the previously proposed probabilistic event-based scheduler [10] to a bi-character rule which possesses both probabilistic and deterministic features. In [10] we show that under an emulative control law, the pure probabilistic scheduling rule is stabilizing for an NCS of LTI local systems with ideal communication channels. Here we analyze stability and investigate robustness of the modified access protocol for multi-loop NCSs with the local loops coupled through a shared non-ideal network subject to limited capacity, delay and packet dropouts. The present policy enhances the performance of NCSs by keeping the subsystems with insignificant error levels out of the channel access competition. Subsequently, the medium access is granted to the transmission requests in a probabilistic manner. In an ideal situation, the scheduler collects the local data from each control loop in order to decide the transmission order. In reality, the sheer amount of information may prevent the scheduler from processing all these information at once, which induces delays. Even though the scheduler might be fed with delayed transmission requests, the local controllers are updated (provided no dropouts occur) with the latest state values and without delays. Notice that the error-dependent 
scheduler does not need to know the state vector to decide the access order, but only the norm of error. In addition, we show that the stochastic nature of our scheduling policy provides flexibility to cope with packet dropouts while preserving stability. Moreover, the randomization in the scheduling process facilitates an approximative decentralized implementation.

In the remainder of this paper, Section II states the problem of interest and some preliminaries regarding stability of Markov chains. In Section III, we analyze stability of the resulting Markov chain under channel imperfections. Simulations and numerical results are shown in Section IV. Notation Euclidean norm and conditional expectation are denoted $\|\cdot\|_{2}$ and $\mathrm{E}[\cdot \mid \cdot]$, respectively. $\mathcal{N}(\mu, X)$ represents the Gaussian distribution with mean $\mu$ and covariance matrix $X$. A state vector $x_{k}^{i}$ belongs to control loop $i$, with subscript $k$ denoting the time. For matrices, the subscript indicates the corresponding loop and superscript denotes the matrix power.

\section{PROBLEM STATEMENT AND PRELIMINARIES}

We consider an NCS composed of $N$ heterogeneous LTI control loops coupled through a non-ideal shared communication network, as depicted in Fig. 1. Each individual loop consists of a linear plant $\mathcal{P}^{i}$ and a stabilizing controller $\mathcal{C}^{i}$. An event-based scheduler decides if a state vector $x_{k}^{i} \in \mathbb{R}^{n_{i}}$ is an event to be scheduled for channel access. The plant $\mathcal{P}^{i}$ is modeled by the following stochastic difference equation

$$
x_{k+1}^{i}=A_{i} x_{k}^{i}+B_{i} u_{k}^{i}+w_{k}^{i},
$$

where $w_{k}^{i} \in \mathbb{R}^{n_{i}}$ is i.i.d. with $\mathcal{N}(0, I)$ while $A_{i} \in \mathbb{R}^{n_{i} \times n_{i}}$ and $B_{i} \in \mathbb{R}^{n_{i} \times m_{i}}$ describe system and input matrices, respectively. The initial state $x_{0}^{i}$ is chosen randomly from an arbitrary bounded-variance distribution. At each time-step $k$, the variable $\delta_{k}^{i}$ denotes the scheduler's decision for loop $i$ as

$$
\delta_{k}^{i}= \begin{cases}1, & x_{k}^{i} \text { sent through the channel } \\ 0, & x_{k}^{i} \text { blocked. }\end{cases}
$$

However, data packets that are awarded the channel access might drop due to transmission errors in the network links or congestion. A successful data packet transmission is confirmed upon the packet arrival via the variable $\gamma_{k}^{i} \in\{0,1\}$

$$
\gamma_{k}^{i}= \begin{cases}1, & x_{k}^{i} \text { successfully received } \\ 0, & x_{k}^{i} \text { dropped. }\end{cases}
$$

Accordingly, the controller $u_{k}^{i}$ is updated with the true state values $x_{k}^{i}$ only if $\delta_{k}^{i}=1 \wedge \gamma_{k}^{i}=1$.

We employ an emulation-based control strategy with the minimum required assumptions, i.e. stabilizing linear control laws. Assuming that the $i^{\text {th }}$ loop has local knowledge $A_{i}, B_{i}$ and the distribution of $x_{0}^{i}$, the control law $\vartheta^{i}$ is described by

$$
u_{k}^{i}=\vartheta_{k}^{i}\left(Z_{k}^{i}\right)=-L_{i} \mathrm{E}\left[x_{k}^{i} \mid Z_{k}^{i}\right]
$$

where $L_{i}$ is any arbitrary stabilizing feedback gain and $Z_{k}^{i}=$ $\left\{x_{0}^{i}, \delta_{0}^{i}, \ldots, x_{k-1}^{i}, \delta_{k-1}^{i}\right\}$ is the controller's update history. In case no new information is received, $\hat{x}_{k}^{i}$ is computed by a Kalman-like estimator

$$
\mathrm{E}\left[x_{k}^{i} \mid Z_{k}^{i}\right]=\left(A_{i}-B_{i} L_{i}\right) \mathrm{E}\left[x_{k-1}^{i} \mid Z_{k-1}^{i}\right],
$$

with the initial condition $\mathrm{E}\left[x_{0}^{i} \mid Z_{0}^{i}\right]=0$. Accordingly, the network-induced estimation error $e_{k}^{i} \in \mathbb{R}^{n_{i}}$ is defined as $e_{k}^{i}=$ $x_{k}^{i}-\mathrm{E}\left[x_{k}^{i} \mid Z_{k}^{i}\right]$ at each time $k$. Employing (1)-(3), we have

$$
e_{k+1}^{i}=\left(1-\theta_{k+1}^{i}\right) A_{i} e_{k}^{i}+w_{k}^{i},
$$

where $\theta_{k}^{i}=\delta_{k}^{i} \gamma_{k}^{i}$. It is worth mentioning that the ordering of decisions within one time period is assigned by the sequence

$$
\cdots \rightarrow e_{k} \rightarrow \delta_{k+1} \rightarrow \theta_{k+1} \rightarrow u_{k+1} \rightarrow e_{k+1} \rightarrow \cdots
$$

The aggregate state $\left[x_{k}^{i \top}, e_{k}^{i \top}\right]^{\top}$ has a triangular dynamics (see [10] for more) for each subsystem according to (1)-(4), which in turn confirms that the evolution of $e_{k}^{i}$ is independent of $x_{k}^{i}$. This implies that it is sufficient to show stability of $e_{k}$ in order to show the overall networked system stability.

Assuming not all subsystems can simultaneously transmit, the following bi-character error-dependent rule defines the channel access probability for each subsystem at time $k+1$ according to a deterministic-probabilistic measure:

$\mathrm{P}\left[\delta_{k+1}^{i}=1 \mid e_{k}^{j}, \lambda_{j}\right]= \begin{cases}0, & \left\|e_{k}^{i}\right\|_{Q^{i}}^{2} \leq \lambda_{i} \\ 1, & \left\|e_{k-d_{k}^{i}}^{i}\right\|_{Q^{i}}^{2}>\lambda_{i} \wedge j_{\lambda} \leq c \\ \frac{\left\|e_{k-d_{k}^{i}}^{i}\right\|_{Q^{i}}^{2}}{\sum_{j_{\lambda} k_{k-d_{k}^{j}}\left\|e_{Q^{j}}\right\|^{2}}^{2}}, & \left\|e_{k-d_{k}^{i}}^{i}\right\|_{Q^{i}}^{2}>\lambda_{i} \wedge j_{\lambda}>c\end{cases}$

where $\lambda_{i}$ 's represent the local error thresholds for subsystems $i \in\{1, \ldots, N\}, d_{k}^{i}$ denotes the delay in transmission request submission at time $k$, and $Q^{i}$, s are symmetric positive definite weight matrices. Moreover, $j_{\lambda}$ denotes the number of qualified subsystems for access competition, and $c<N$ denotes the channel capacity whilst $\left\|e_{k}^{j}\right\|_{Q_{j}}^{2}:=e_{k}^{j \top} Q_{j} e_{k}^{j}$.

To clarify further analyses, it should be noted that there exist two different types of communication channels for data transmission in our NCSs. One is the shared communication channel over which the subsystems send their latest state values to their corresponding controllers (if they are awarded the access by the scheduler). This channel is subject to the capacity constraint $c<N$, as well as the possibility that the scheduled data packets are dropped when passing through. However, we assume that this channel is delayfree, i.e. if a subsystem is awarded the access at some time $k^{\prime}$, and if the data packet is not dropped (i.e. $\theta_{k^{\prime}}^{i}=1$ ), then the $i^{\text {th }}$ controller will be immediately updated with the latest state values. There is, however, another communication channel between the subsystems and the scheduling unit over which the local subsystems submit their transmission requests to the scheduler. This channel does not have capacity constraints as the amount of data being exchanged over this channel is much lower than the information load over the former communication channel. The latter channel, however, is subject to time-varying delay $d_{k^{\prime}}^{i}$. Thus, a transmission request from a local system $i$, which is supposed to be considered for access competition at time step $k^{\prime}$, is received by the scheduler with some time-variant and finite delay $d_{k^{\prime}}^{i}$. Consequently, only the scheduling policy (5) is affected by the delays and not the difference equation (4). 


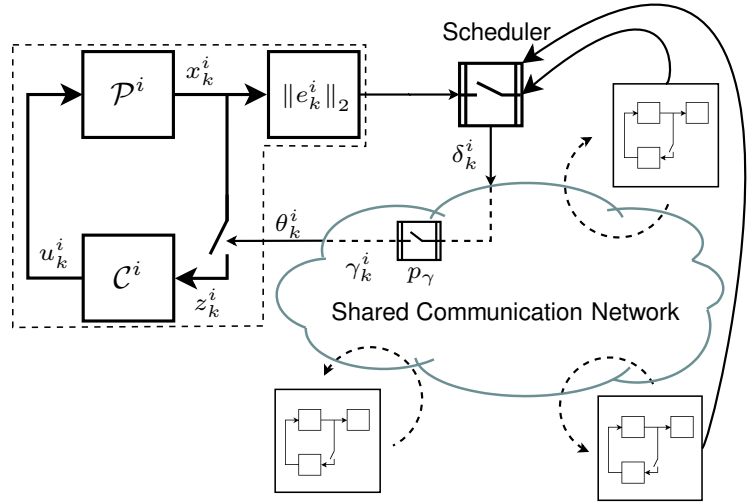

Fig. 1. A multi-loop NCS with shared communication channel.

As the local errors are known to their corresponding loops, the first argument of policy (5) is checked locally within each subsystem to decide whether a transmission request should be submitted. Therefore, a transmission request from subsystem $i$ is submitted at time-step $k$ to the scheduler only if $\left\|e_{k}^{i}\right\|_{Q^{i}}^{2}>\lambda_{i}$. Depending on the delay time $d_{k}^{i}$, the scheduler takes the channel access request into account either in the same time step (in case $d_{k}^{i}=0$ ) or later on (in case $d_{k}^{i} \neq 0$ ). It can be seen from (5) that only the norm of error is required for deciding on channel access probability, and not the whole state vector. Therefore, as far as a subsystem is awarded the channel access, either from a transmission request at that current time-step or from a delayed request, the corresponding controller will be updated with the latest state vector. If $j_{\lambda} \leq c$, then all of the received requests will transmit as seen from the second argument of (5). Otherwise, the channel is allocated probabilistically until the capacity is reached, while the other transmission requests are blocked. In the interest of paper brevity, we assume $c=1$, i.e.

$$
\sum_{i=1}^{N} \delta_{k}^{i}=1 .
$$

The following results can straightforwardly be extended towards $\sum_{i=1}^{N} \delta_{k}^{i}=c<N$.

Remark 1: According to (5), the design parameters $\lambda_{i}$ and $Q^{i}$ appear in the deterministic and probabilistic parts of the scheduling process, respectively. The former represents the threshold that the $i^{\text {th }}$ subsystem can tolerate until it becomes qualified to compete for channel access, and $Q^{i}$ specifies how frequently the $i^{\text {th }}$ subsystem needs data transmission.

We define the aggregate error state $e_{k} \in \mathbb{R}^{n}$ as follows:

$$
e_{k}=\left[e_{k}^{1 \top}, \ldots, e_{k}^{N \top}\right]^{\top},
$$

where $n=\sum_{i=1}^{N} n_{i}$. The scheduling rule in (5) is a randomized policy depending on the latest received error values. Therefore, (7) is a Markov chain. Moreover, as the difference equation in (4) is time-invariant and the noise process $w_{k}^{i}$ is i.i.d. for $i \in\{1, \ldots, N\}$, the Markov chain (7) is homogeneous. Since the noise distribution is absolutely continuous having a positive density function, it is furthermore concluded that the chain is aperiodic and $\psi$-irreducible.

\section{A. Decentralized Implementation of the Scheduler}

The stochastic nature of the policy (5) enables a decentralized implementation within the CSMA protocol. As the details of implementation go beyond the scope of this paper, we focus on sketching the main idea of the protocol.

The CSMA model follows the assumptions: (i) sensing the carrier is instantaneous, (ii) there are no hidden nodes, (iii) the backoff intervals are exponentially distributed with errordependent exponents, (iv) the mean backoff time is negligible compared to the sampling interval, (v) data packets are discarded after one retransmission trial. The assumptions (i) and (ii) rule out the packet collisions. The assumptions (iii)-(v) are tailored to the discrete-time nature of the control process. Moreover, assumption (vi) guarantees that the transmission is accomplished at the end of each sampling interval.

At the beginning of every sampling instance, each eligible subsystem waits to transmit according to the randomly chosen backoff interval, according to assumption (iii) and depends on the current error of the subsystem. The subsystem with the smallest interval is permitted to transmit, while all other subsystems are blocked. Furthermore, the mean backoff interval decreases with increasing error norm. This naturally leads to a prioritization of the control loops.

\section{Stability Analysis}

In this section, stability of a multi-loop NCS with shared communication resource subject to capacity constraint (6) and channel uncertainties, scheduled by the policy (5) is investigated. Stability is shown in terms of $f$-ergodicity of the Markov chain $e_{k}$ (7).

\section{A. Preliminaries}

A random process is called ergodic if the time-average of its events over one sample sequence of transitions represents the process behavior over the entire state-space. Thus, ergodicity implies that an invariant finite measure exists over the entire state-space, such that the process returns to some sets in finite time, and does not diverge forever.

Definition 1: [17] Let $f \geq 1$ be a real valued function in $\mathbb{R}^{n}$. A Markov chain $\Phi$ is said to be $f$-ergodic, if

1) $\Phi$ is positive Harris recurrent with the unique invariant probability measure $\pi$

2) the expectation $\pi(f):=\int f\left(\Phi_{k}\right) \pi\left(d \Phi_{k}\right)$ is finite

3) $\lim _{k \rightarrow \infty}\left\|P^{k}\left(\Phi_{0}, .\right)-\pi\right\|_{f}=0$ for every initial value $\Phi_{0} \in X$, where $\|\nu\|_{f}=\sup _{|g| \leq f}|\nu(g)|$.

Definition 2 (Drift operator): Let $V: \mathbb{R}^{n} \rightarrow \mathbb{R}^{+}$be a realvalued function and $\Phi$ be a Markov chain. The drift operator $\Delta$ is defined for any non-negative measurable function $V$ as

$$
\Delta V\left(\Phi_{k}\right)=\mathrm{E}\left[V\left(\Phi_{k+1}\right) \mid \Phi_{k}\right]-V\left(\Phi_{k}\right), \quad \Phi_{k} \in \mathbb{R}^{n} .
$$

The following theorem summarizes the $f$-ergodicity of Markov chains in general state spaces [17, Ch. 14].

Theorem 1 ( $f$-Norm Ergodic): Let the Markov chain $\Phi$ be $\psi$-irreducible and aperiodic and $f \geq 1$ be a real-valued function in $\mathbb{R}^{n}$. If a small set $\mathcal{D}$ and a non-negative realvalued function $V$ exists such that $\Delta V(\Phi) \leq-f(\Phi)$ for any $\Phi \in \mathbb{R}^{n} \backslash \mathcal{D}$ and $\Delta V<\infty$ for $\Phi \in \mathcal{D}, \Phi$ is $f$-ergodic. 
Remark 2: Provided that a linear state-space model is $\psi$ irreducible, all compact subsets of the state space are small sets [17, Sec. 5.3.5].

Let us select the following Lyapunov function $V: \mathbb{R}^{n} \rightarrow \mathbb{R}^{+}$:

$$
V\left(e_{k}\right)=\sum_{i=1}^{N} e_{k}^{i^{\top}} Q_{k}^{i} e_{k}^{i}:=\sum_{i=1}^{N}\left\|e_{k}^{i}\right\|_{Q^{i}}^{2}
$$

Due to properties of the selected function (9), $f$-ergodicity of the Markov chain (7) is not always guaranteed employing the drift $\Delta V$ over one transition step, i.e. $k \rightarrow k+1$. We show this for $N=2$ by constructing the following example.

\section{B. Illustrative example}

Let an NCS of two identical scalar systems competing for the sole channel slot. For simplicity, assume $Q^{1}=Q^{2}=I$ and $e_{k}^{1}=e_{k}^{2}=\bar{e}_{k}>\lambda_{1}=\lambda_{2}$, so the access chance for each system is $\frac{1}{2}$ according to (5). In addition, assume no dropout happens and $d_{k}^{i}=0$. From (8) with $e_{k}=\left[e_{k}^{1} e_{k}^{2}\right]^{\top}$, we have

$$
\begin{aligned}
& \Delta V\left(e_{k}\right)=\mathrm{E}\left[V\left(e_{k+1}\right) \mid e_{k}\right]-V\left(e_{k}\right) \\
& \quad=\frac{1}{2} \sum_{i=1,2} \mathrm{E}\left[\left\|w_{k}^{i}\right\|_{I}^{2}\right]+\mathrm{E}\left[\left\|A \bar{e}_{k}+w_{k}^{i}\right\|_{I}^{2} \mid e_{k}\right]-2\left\|\bar{e}_{k}\right\|_{I}^{2} \\
& \quad=2+\left\|A \bar{e}_{k}\right\|_{I}^{2}-2\left\|\bar{e}_{k}\right\|_{I}^{2},
\end{aligned}
$$

For $A>\sqrt{2}$, the drift is positive, which violates the condition in Theorem 1 on a non-compact set. Now, we compute the drift over two steps $[k, k+2]$. Assuming that the first subsystem transmits at time $k+1$, i.e. $e_{k+1}^{1}=w_{k}^{1}$ we have

$$
\begin{aligned}
& \Delta V\left(e_{k}, 2\right)=\mathrm{E}\left[V\left(e_{k+2}\right) \mid e_{k}\right]-V\left(e_{k}\right) \\
& =\sum_{i=1,2} \mathrm{E}\left[\left\|\left(1-\delta_{k+2}^{i}\right) A e_{k+1}^{i}+w_{k+1}^{i}\right\|_{I}^{2} \mid e_{k}\right]-2\left\|\bar{e}_{k}\right\|_{I}^{2} \\
& \leq \mathrm{E}\left[\left\|\left(1-\delta_{k+2}^{2}\right) A e_{k+1}^{2}\right\|_{I}^{2} \mid e_{k}\right]+\|A\|_{2}^{2}+2-2\left\|\bar{e}_{k}\right\|_{I}^{2} .
\end{aligned}
$$

According to the law of iterated expectations, we have

$$
\begin{aligned}
& \Delta V\left(e_{k}, 2\right) \leq\|A\|_{2}^{2}+2-2\left\|\bar{e}_{k}\right\|_{I}^{2} \\
& +\mathrm{E}\left[\mathrm{E}\left[\left(1-\delta_{k+2}^{2}\right) \mid e_{k+1}, e_{k}\right]\|A\|_{2}^{2}\left\|e_{k+1}^{2}\right\|_{I}^{2} \mid e_{k}\right] \\
& \leq\|A\|_{2}^{2}+2-2\left\|\bar{e}_{k}\right\|_{I}^{2}+\mathrm{E}\left[\frac{\left\|w_{k}^{1}\right\|_{I}^{2}}{\left\|w_{k}^{1}\right\|_{I}^{2}+\left\|e_{k+1}^{2}\right\|_{I}^{2}}\|A\|_{2}^{2}\left\|e_{k+1}^{2}\right\|_{I}^{2} \mid e_{k}\right] \\
& \leq 2\|A\|_{2}^{2}+2-2\left\|\bar{e}_{k}\right\|_{I}^{2} .
\end{aligned}
$$

Define $f\left(e_{k}\right)=2\left[\varepsilon\left\|\bar{e}_{k}\right\|_{I}^{2}-\|A\|_{2}^{2}-1\right]$ with $\varepsilon \in(0,1]$, we can find small set $\mathcal{D}$ such that for $e_{k} \in \mathbb{R}^{n} / \mathcal{D}, f \geq 1$, and $\Delta V \leq-f$. In addition, $\Delta V<\infty$ for $e_{k} \in \mathcal{D}$, and Theorem 1 holds.

It concludes only after all subsystems have a chance to transmit, a negative drift over some interval can be guaranteed. To fulfill this, we investigate the ergodicity of the Markov chain over an interval with length $N$. It is worth noting that ergodicity over an interval implies ergodicity over longer intervals [18]. To infer the $f$-ergodicity over the interval $[k, k+N]$, we modify the drift definition (8) as

$$
\Delta V\left(e_{k}, N\right)=\mathrm{E}\left[V\left(e_{k+N}\right) \mid e_{k}\right]-V\left(e_{k}\right), \quad e_{k} \in \mathbb{R}^{n} .
$$

To investigate the stability and robustness, we consider two phenomena: delay in transmission request submission (over the channel between subsystems and the scheduler) to the scheduler, and packet dropouts in the limited capacity communication channel (to update the controllers).

\section{Stochastic Stability with Delayed Information Update}

To study stability in the presence of arbitrary time-varying finite delays, we assume that the considered NCS has operated from time $k$ to $k+N-1$ utilizing the policy (5). Then at the last time $k+N$, we let the scheduler decide the channel access considering all scenarios that might have happened over $[k, k+N-1]$. We define at every time $k^{\prime} \in[k, k+N]$ two time-varying disjoint sets of subsystems, $S_{k^{\prime}}^{1}$ and $S_{k^{\prime}}^{2}$, as

$$
i \in\left\{\begin{array}{ll}
S_{k^{\prime}}^{1} & \text { if }\left\|e_{k^{\prime}}^{i}\right\|_{Q^{i}}^{2} \leq \lambda_{i} \\
S_{k^{\prime}}^{2} & \text { if }\left\|e_{k^{\prime}}^{i}\right\|_{Q^{i}}^{2}>\lambda_{i}
\end{array},\right.
$$

with $S_{k^{\prime}}^{1} \cup S_{k^{\prime}}^{2}=N$. The systems included in $S_{k^{\prime}}^{2}$ are eligible for channel access competition at time $k^{\prime}+1$, while $S_{k^{\prime}}^{1}$ contains the excluded systems. Since not only a transmission results in error decrement, but the noise might also decrease the error, the systems' inclusion in either set $S_{k^{\prime}}^{1}$ or $S_{k^{\prime}}^{2}$ depends on both transmission occurrence and noise process. Following the lines of [10], we discern three complementary and mutually exclusive cases for a system $i$ at time $k+N-1$ :

Subsystem $i$ :

$c_{1}$ : has either successfully transmitted or not within the past $N-1$ time-steps, and is in set $S_{k+N-1}^{1}$, i.e.

$$
i \in S_{k+N-1}^{1} \quad \Rightarrow \quad\left\|e_{k+N-1}^{i}\right\|_{Q^{i}}^{2} \leq \lambda_{i},
$$

$c_{2}$ : has successfully transmitted at least once within the past $N-1$ time-steps, and is in set $S_{k+N-1}^{2}$, i.e.

$$
\exists k^{\prime} \in[k, k+N-1]: \theta_{k^{\prime}}^{i}=1 \text { and }\left\|e_{k+N-1}^{i}\right\|_{Q^{i}}^{2}>\lambda_{i},
$$

$c_{3}$ : has not successfully transmitted due to either blocking by the scheduler $\left(\delta_{k^{\prime}}^{i}=0\right)$ or drop out $\left(\gamma_{k^{\prime}}^{i}=0\right)$ within the past $N-1$ time-steps, and is in set $S_{k+N-1}^{2}$, i.e.

$$
\forall k^{\prime} \in[k, k+N-1]: \theta_{k^{\prime}}^{i}=0 \text { and }\left\|e_{k+N-1}^{i}\right\|_{Q^{i}}^{2}>\lambda_{i} .
$$

Each subsystem is characterized by one of the above disjoint cases during $[k, k+N-1]$, and thus the cardinality of the union equals $N$. Before proceeding, we point out that the value of $e_{k+N}$ can be given as a function of previous error values at a certain time-step $k+r^{\prime}-1$, with $r^{\prime} \in[0, N-1]$ :

$$
\begin{aligned}
e_{k+N}^{i} & =\prod_{j=r^{\prime}}^{N}\left(1-\delta_{k+j}^{i}\right) A_{i}^{N-r^{\prime}+1} e_{k+r^{\prime}-1}^{i} \\
& +\sum_{r=r^{\prime}}^{N}\left[\prod_{j=r+1}^{N}\left(1-\delta_{k+j}^{i}\right) A_{i}^{N-r} w_{k+r-1}^{i}\right],
\end{aligned}
$$

where we define $\prod_{N+1}^{N}\left(1-\delta_{k+j}^{i}\right):=1$.

Theorem 2: Let in an NCS of interest the local control loops share a communication channel constrained by (6). Suppose that a transmission request from subsystem $i$ is received by the scheduler with the delay $d_{k}^{i}<N$. Assume the stabilizing control laws are given by (2), and channel access is scheduled by (5), then the Markov chain (7) is $f$-ergodic.

Proof: For now, we assume that the communication channel is perfect i.e. no data is dropped after it is awarded the transmission slot. The $N$-step drift (10) is split into partial drifts for each case $c_{1}, c_{2}$ and $c_{3}$ as

$$
\Delta V\left(e_{k}^{i \in c_{j}}, N\right)=\sum_{c_{j}} \mathrm{E}\left[\left\|e_{k+N}^{i}\right\|_{Q^{i}}^{2} \mid e_{k}\right]-V\left(e_{k}^{c_{j}}\right)
$$


where $V\left(e_{k}^{c_{j}}\right)=\sum_{c_{j}}\left\|e_{k}^{i}\right\|_{Q^{i}}^{2}$. In what follows, we investigate (13) for each case $c_{j}$ to invoke Theorem 1 employing the Lyapunov function (9). Before proceeding, we point out that the conditions of Theorem 1 hold by showing that the expectation of error norm in $N$ steps ahead is bounded. We can show this for the cases $c_{1}$ and $c_{2}$ regardless of the scheduling process. We exploit the fact that for the systems in $c_{1}$ and $c_{2}$ the error is below the corresponding thresholds at certain time-steps. Due to linearity of systems, this suffices to show the boundedness of the error expectation over further intervals with finite lengths. Notice that delays do not impact this inference, but merely the conservativeness of the obtained upper bounds. However, the third case is more involved and delays have to be considered when showing boundedness of the expected error over $N$ steps ahead.

For the first case, the subsystems belong to $S_{k+N-1}^{1}$, which implies $\left\|e_{k+N-1}^{i}\right\|_{Q^{i}}^{2} \leq \lambda_{i}$, no matter if they transmitted or not. This implies $\delta_{k+N}^{i}=0$ for all $i \in c_{1}$. Consequently,

$$
\begin{aligned}
& \sum_{c_{1}} \mathrm{E}\left[\left\|e_{k+N}^{i}\right\|_{Q^{i}}^{2} \mid e_{k}\right]=\sum_{c_{1}} \mathrm{E}\left[\left\|A_{i} e_{k+N-1}^{i}+w_{k+N-1}^{i}\right\|_{Q^{i}}^{2} \mid e_{k}\right] \\
& \leq \sum_{c_{1}}\left\|A_{i}\right\|_{2}^{2} \mathrm{E}\left[\left\|e_{k+N-1}^{i}\right\|_{Q^{i}}^{2} \mid e_{k}\right]+\mathrm{E}\left[\left\|w_{k+N-1}^{i}\right\|_{Q^{i}}^{2}\right],
\end{aligned}
$$

where the inequality is ensured by the Cauchy-Schwarz inequality. Since $\left\|e_{k+N-1}^{i}\right\|_{Q^{i}}^{2} \leq \lambda_{i}$, it concludes

$$
\sum_{c_{1}} \mathrm{E}\left[\left\|e_{k+N}^{i}\right\|_{Q^{i}}^{2} \mid e_{k}\right] \leq \sum_{c_{1}}\left\|A_{i}\right\|_{2}^{2} \lambda_{i}+\mathrm{E}\left[\left\|w_{k+N-1}^{i}\right\|_{Q^{i}}^{2}\right] .
$$

Having the partial drift (13), we define $f_{c_{1}}=\epsilon_{1} V\left(e_{k}^{c_{1}}\right)-\xi_{1}^{b^{+}}$, where $\xi_{1}^{b^{+}}$stands for the upper bound (14) and $\epsilon_{1} \in(0,1]$. Since compact sets of $\mathbb{R}^{n}$ are all small, we can find small set $\mathcal{D}_{1}$ and $\epsilon_{1}$ such that $f_{c_{1}} \geq 1$ and $\Delta V\left(e_{k}^{c_{1}}, N\right) \leq-f_{c_{1}}$.

For $i \in c_{2}$, assume a transmission has occurred at time $k+r_{i}^{\prime}$, where $r_{i}^{\prime} \in[0, N-1]$, i.e. $\delta_{k+r_{i}^{\prime}}^{i}=1$. Statistical independence of $w_{k+r}^{i}$ and error $e_{k+r_{i}^{\prime}-1}^{i}$ yields from (12)

$$
\begin{aligned}
& \sum_{c_{2}} \mathrm{E}\left[\left\|e_{k+N}^{i}\right\|_{Q^{i}}^{2} \mid e_{k}\right] \\
& =\sum_{c_{2}} \sum_{r=r_{i}^{\prime}}^{N} \mathrm{E}\left[\prod_{j=r+1}^{N}\left[1-\delta_{k+j}^{i}\right]\right] \mathrm{E}\left[\left\|A_{i}^{N-r} w_{k+r-1}^{i}\right\|_{Q^{i}}^{2}\right] \\
& \leq \sum_{c_{2}} \sum_{r=r_{i}^{\prime}}^{N} \mathrm{E}\left[\left\|A_{i}^{N-r} w_{k+r-1}^{i}\right\|_{Q^{i}}^{2}\right]
\end{aligned}
$$

Define $f_{c_{2}}=\epsilon_{2} V\left(e_{k}^{c_{2}}\right)-\xi_{2}^{b^{+}}$, where $\xi_{2}^{b^{+}}$is given in (15) and $\epsilon_{2} \in(0,1]$. Then we can find a small set $\mathcal{D}_{2} \subset \mathbb{R}^{n}$ and constant $\epsilon_{2}$ such that $f_{c_{2}} \geq 1$ and $\Delta V\left(e_{k}^{c_{2}}, N\right) \leq-f_{c_{2}}$.

The subsystems in the third case are eligible for channel access in final time $k+N$, while they have never transmitted. Recall that, due to the probabilistic feature of our scheduler, there is no guarantee that a system with higher priority for channel access wins the competition against one with lower priority. To infer $f$-ergodicity in this case, we split the third case $c_{3}$ into two complementary and disjoint sub-cases:

$l_{1}^{c_{3}}$ Subsystem $i$ has not transmitted within the past $N-1$ time steps, but $i \in S^{1}$ at least once, with the last inclusion occurred at a time $k+r_{i}^{\prime}$, with $r_{i}^{\prime} \in[0, \ldots, N-2]$, $l_{2}^{c_{3}}$ Subsystem $i$ has not transmitted within the past $N-1$ time-steps, and $i \in S^{2}$ for all time steps $[k, k+N-1]$.

Recall that for $i \in c_{3}$, we have $i \in S_{k+N-1}^{2}$. For a subsystem $i \in l_{1}^{c_{3}}$, suppose that $i \in S_{k+r_{i}^{\prime}}^{1}$, which implies $\left\|e_{k+r_{i}^{\prime}}^{i}\right\|_{Q^{i}}^{2} \leq \lambda_{i}$. Knowing for $i \in c_{3}, \delta_{k^{\prime}}^{i}=0$ for $k^{\prime} \in[k, k+N-1]$, we reach

$$
\begin{aligned}
& \sum_{l_{1}^{c_{3}}} \mathrm{E}\left[\left\|e_{k+N}^{i}\right\|_{Q^{i}}^{2} \mid e_{k}\right] \leq \\
& \sum_{l_{1}^{c_{3}}}\left[\left\|A_{i}^{N-r_{i}^{\prime}}\right\|_{2}^{2} \lambda_{i}+\sum_{r=r_{i}^{\prime}}^{N-1} \mathrm{E}\left[\left\|A_{i}^{N-r-1} w_{k+r}^{i}\right\|_{Q^{i}}^{2}\right]\right]
\end{aligned}
$$

Define $f_{l_{1}^{c_{3}}}=\epsilon_{l_{1}^{c_{3}}} V\left(e_{k}^{l_{1}^{c_{3}}}\right)-\xi_{l_{1}^{c_{3}}}^{b^{+}}$, where $\xi_{l_{1}^{c_{3}}}^{b^{+}}$stands for the expression (16), with $\epsilon_{l_{1}^{c_{3}}} \in(0,1]$. Thus, we can find small set $\mathcal{D}_{l_{1}^{c_{3}}}$ and $\epsilon_{l_{1}^{c_{3}}}$ such that $f_{l_{1}^{c_{3}}} \geq 1$, and $\Delta V\left(e_{k}^{l_{1}^{c_{3}}}, N\right) \leq-f_{l_{1}^{c_{3}}}$.

In sub-case $l_{2}^{c_{3}}$, we know $\left\|e_{k^{\prime}}^{i}\right\|_{Q_{i}}^{2}>\lambda_{i}$ for all time steps $k^{\prime} \in[k, k+N-1]$. Considering (12) with $r^{\prime}=0$, we have

$$
\begin{aligned}
& \sum_{l_{2}^{c_{3}}} \mathrm{E}\left[\left\|e_{k+N}^{i}\right\|_{Q^{i}}^{2} \mid e_{k}\right] \\
& \leq \sum_{l_{2}^{c_{3}}} \mathrm{E}\left[\left\|A_{i}^{N} e_{k}^{i}+\sum_{1}^{N}\left[A_{i}^{N-r} w_{k+r-1}^{i}\right]\right\|_{Q^{i}}^{2} \mid e_{k}\right] \\
& \leq \sum_{l_{2}^{c_{3}}} \mathrm{E}\left[\left\|\sum_{1}^{N}\left[A_{i}^{N-r} w_{k+r-1}^{i}\right]\right\|_{Q^{i}}^{2}\right]+\left\|A_{i}^{N}\right\|_{2}^{2}\left\|e_{k}^{i}\right\|_{Q^{i}}^{2} \\
& \leq \sum_{l_{2}^{c_{3}}} \mathrm{E}\left[\left\|\sum_{1}^{N}\left[A_{i}^{N-r} w_{k+r-1}^{i}\right]\right\|_{Q^{i}}^{2}\right]+\left\|A_{i}^{N}\right\|_{2}^{2} V\left(e_{k}^{l_{2}^{c_{3}}}\right)
\end{aligned}
$$

Dependence of (17) on the initial value via the last term implies that we cannot find an appropriate $f_{l_{2}^{c_{3}}}$ to invoke Theorem 1 for arbitrary initial value. However, as the considered cases are mutually exclusive, we calculate the probability for sub-case $l_{2}^{c_{3}}$ to happen according to the scheduling policy (5). Let one system, say $j$, does not transmit during the interval $[k, k+N-1]$, then there exists another subsystem, say $i$, which is awarded the channel access more than once. Assume that the scheduler is to decide the channel access at the final time step $k+N$, while $e_{k+N-d_{k+N-1}^{i}-1}^{i}$ is the latest information the scheduler receives of subsystem $i$, with $d_{k+N-1}^{i}<N$. Recall that the subsystems in the set $c_{1}$ are not eligible for transmission, thus $i \in c_{2}$. We consider the worst-case scenario by assuming that the subsystems included in $l_{2}^{c_{3}}$ satisfy $\left\|e_{k^{\prime}}^{j}\right\|_{Q^{j}}^{2} \leq\left\|e_{k^{\prime}+1}^{j}\right\|_{Q^{j}}^{2}$. In fact, the worst case scenario considers $j \in l_{2}^{c_{3}}$ with their respective errors monotonically increasing from one time-step to the next. Assume $k+\bar{r}<k+N$ is the last time-step the subsystem $i \in c_{2}$ has transmitted i.e. $\delta_{k+\bar{r}}^{i}=1$. Thus, the probability of happening $l_{2}^{c_{3}}$ considering delayed information update is

$$
\begin{aligned}
& \mathrm{P}\left[\delta_{k+N}^{i}=1 \mid \delta_{k+\bar{r}}^{i}=1, d_{k+N-1}^{j},\left\|e_{\bar{k}}^{j}\right\|_{Q^{j}}^{2}>\lambda_{j}\right] \\
& =\mathrm{E}\left[\frac{\left\|e_{k+k_{d_{i}}}^{i}\right\|_{Q^{i}}^{2}}{\sum_{i \in c_{2}}\left\|e_{k+k_{d_{i}}}^{i}\right\|_{Q^{i}}^{2}+\sum_{j \in c_{3}}\left\|e_{k+k_{d_{j}}}^{j}\right\|_{Q^{j}}^{2}} \mid z_{i, j}\right] \\
& \leq \mathrm{E}\left[\frac{\left\|\sum_{r=\bar{r}}^{N-2} A_{i}^{k_{d_{i}}-r} w_{k+r}^{i}\right\|_{Q^{i}}^{2}}{\sum_{c_{2}} \lambda_{i}+\sum_{l_{1}^{c_{3}}}\left\|e_{k+k_{d_{j}}}^{j}\right\|_{Q^{j}}^{2}+\sum_{l_{2}}^{c_{3}}\left\|e_{k+k_{d_{j}}}^{j}\right\|_{Q^{j}}^{2}} \mid z_{i, j}\right] \\
& \leq \frac{\sum_{r=\bar{r}}^{N-2} \mathrm{E}\left[\left\|A_{i}^{k_{d_{i}}-r} w_{k+r}^{i}\right\|_{Q^{i}}^{2}\right]}{\sum_{c_{2}} \lambda_{i}+\sum_{l_{1}^{c_{3}}} \lambda_{j}+\sum_{l_{2}}^{c_{3}}\left\|e_{k}^{j}\right\|_{Q^{j}}^{2}}=\mathrm{P}_{l_{2}^{c_{3}}} .
\end{aligned}
$$


where $k_{d_{i}}=N-d_{k+N-1}^{i}-1$, and $z_{i, j}$ stands for the conditions of the expectation. The last expression follows from (11). From (18) one infers that the probability of subsequent transmission for a certain system, in the presence of other competitors with large errors and without prior transmissions, can be made arbitrarily close to zero by tuning $\lambda_{j}$ s and $Q^{j}$ s.

The overall $N$-step drift defined in (10) can be expressed in terms of the partial drifts and their probabilities as

$$
\begin{aligned}
& \Delta V\left(e_{k}, N\right)=\sum_{c_{j}} \mathrm{P}_{c_{j}} \Delta V\left(e_{k}^{i \in c_{j}}, N\right) \\
& \leq \Delta V\left(e_{k}^{c_{1}}, N\right)+\Delta V\left(e_{k}^{c_{2}}, N\right)+\Delta V\left(e_{k}^{l_{1}^{c_{3}}}, N\right)+\mathrm{P}_{l_{2}^{c_{3}}} \Delta V\left(e_{k}^{l_{2}^{c_{3}}}, N\right) \\
& \leq-\left[f_{c_{1}}+f_{c_{2}}+f_{l_{1}^{c_{3}}}+f_{l_{2}^{c_{3}}}\right]=-f\left(e_{k}\right) .
\end{aligned}
$$

where, $\mathrm{P}_{c_{j}}$ represents the probability of case $c_{j}$ being happened, such that $\sum_{c_{j}} \mathrm{P}_{c_{j}}=1$. For sub-case $l_{2}^{c_{3}}$, we have

$$
\mathrm{P}_{l_{2}^{c_{3}}} \Delta V\left(e_{k}^{l_{2}^{c_{3}}}, N\right) \leq \mathrm{P}_{l_{2}^{c_{3}}}\left[\left\|A_{j}^{N}\right\|_{2}^{2}-1\right] V\left(e_{k}^{l_{2}^{c_{3}}}\right)+\xi_{l_{2}^{c_{3}}}^{b^{+}},
$$

where $\xi_{l_{2}^{c_{3}}}^{b^{+}}=\mathrm{P}_{l_{2}^{c_{3}}} \sum_{l_{2}^{c_{3}}} \mathrm{E}\left[\left\|\sum_{r=1}^{N} A_{j}^{N-r} w_{k+r-1}^{j}\right\|_{Q^{j}}^{2}\right]$. Define $f_{l_{2}^{c_{3}}}^{c_{3}}=\epsilon_{l_{2}^{c_{3}}} V\left(e_{k}^{l_{2}^{c_{3}}}\right)-\xi_{l_{2}^{c_{3}}}^{b^{+}}$, with $\epsilon_{l_{2}^{c_{3}}} \in(0,1]$. We can find small set $\mathcal{D}_{l_{2}^{c_{3}}}$ and $\epsilon_{l_{2}^{c_{3}}}$ such that $f_{l_{2}^{c_{3}}} \geq 1$, and $\Delta V\left(e_{k}^{l_{2}^{c_{3}}}, N\right) \leq-f_{l_{2}^{c_{3}}}$.

As all partial drifts for cases $c_{j}$ are shown to be finite when accompanied by their probabilities, we can find small set $\mathcal{D}_{f}$ and $\epsilon_{f} \in(0,1]$ such that $f\left(e_{k}\right) \geq 1$, and $\Delta V\left(e_{k}, N\right) \leq$ $-f\left(e_{k}\right)$. This confirms that Theorem 1 holds, and the $f$ ergodicity of Markov chain (7) is followed. In case $d_{k^{\prime}}^{i} \geq N$, i.e. the error remains unknown to the scheduler for the entire interval $[k, k+N], f$-ergodicity of the Markov chain cannot be shown over $[k, k+N]$. Instead, one can extend the length of interval to $2 N, 3 N$, etc. to show the ergodicity, assuming $d_{k^{\prime}}^{i}$ is finite.

It is worth observing how the probability (18) changes with varying delay time as it depends on the stability of the local plant $\mathcal{P}^{i}$. If $\mathcal{P}^{i}$ is stable, then longer delay results in larger values in the numerator. Thus, we need larger error thresholds $\lambda_{i}$ for $i \in c_{2}$ and $\lambda_{j}$ for $j \in l_{1}^{c_{3}}$, in comparison with $d_{k^{\prime}}^{i}=0$, in the denominator to make $\mathrm{P}_{l_{2}^{c_{3}}}$ arbitrarily close to zero. This observation is expected as a stable plant often has lower chance to access the channel in the presence of subsystems with unstable plants and no prior transmission.

\section{Stochastic Stability with Packet Dropouts}

As already stated, the shared communication channel over which the subsystems send their data to their corresponding controllers, is delay-free. Although, the centralized structure of our scheduler implies a collision-free scheme, the consideration of dropouts makes it feasible to handle the collisions while implementing the scheduler in decentralized fashion. So far in the paper, we assumed that data packets which are awarded the channel access are successfully received by the controller. In what follows, we show robustness of the scheduling policy (5) regarding the possibility of dropouts.

Theorem 3: Let an NCS of interest experience $m$ packet dropouts during the interval $[k, k+N]$ in the communication channel. Given the access policy (5), we can find appropriate $\lambda_{i}$ and $Q^{i}$ such that the Markov chain (7) is $f$-ergodic if $m<N$.

Proof: As we discussed in proof of Theorem 2, for the cases $c_{1}, c_{2}$ and $l_{1}^{c_{3}}$ the drift can be proved to be negative ignoring the scheduling process. Thus, packet dropouts do not violate the conditions of Theorem 1 in those cases.

Now assume that subsystem $i \in l_{2}^{c_{3}}$ is awarded the channel access by the scheduler, but the data packet is dropped out. The idea to employ Theorem 1 is as follows - whenever a dropout occurs, we assume that a virtual loop is successfully transmitted instead of a real loop. This means at that timestep, $N$ real and one virtual systems share the communication channel while the channel access is awarded to the latter. The virtual loops have the same discrete LTI dynamics as in (1). Let the channel experience $m<N-1$ dropouts over the interval $[k, k+N-1]$ ensuring at least one successful transmission. Thus, at time $k+N$ we have $N$ real and $m$ virtual systems. The probability that sub-case $l_{2}^{c_{3}}$ occurs is

$$
\begin{aligned}
& \mathrm{P}\left[\delta_{k+N+m}^{i}=1 \mid \delta_{k+\bar{r}}^{i}=1, e_{k}^{j}, m,\left\|e_{\bar{k}}^{j}\right\|_{Q^{j}}^{2}>\lambda_{j}\right] \\
& =\mathrm{E}\left[\frac{\left\|e_{k+k_{d_{m}}}^{i}\right\|_{Q^{i}}^{2}}{\sum_{j \in S^{2}}\left\|e_{k+k_{d_{m}}}^{j}\right\|_{Q^{j}}^{2}} \mid e_{k}^{j}, m, \delta_{k+\bar{r}}^{i}=1,\left\|e_{\bar{k}}^{j}\right\|_{Q^{j}}^{2}>\lambda_{j}\right] \\
& \leq \frac{\sum_{r=\bar{r}}^{N+m-1} \mathrm{E}\left[\left\|A_{i}^{N-r} w_{k+r-1}^{i}\right\|_{Q^{i}}^{2}\right]}{\sum_{j \in c_{2}} \lambda_{j}+\sum_{j \in l_{1}^{c_{3}}} \lambda_{j}+\sum_{j \in l_{2}^{c_{3}}}\left\|e_{k}^{j}\right\|_{Q^{j}}^{2}}=\tilde{\mathrm{P}}_{l_{2}^{c_{3}}}
\end{aligned}
$$

where, $k_{d_{m}}=N+m-1$. The last line holds considering $\left\|e_{k^{\prime}}^{l_{2}^{c_{3}}}\right\|_{Q^{j}}^{2} \leq\left\|e_{k^{\prime}+1}^{l_{2}^{c_{3}}}\right\|_{Q^{j}}^{2}$ for all $k^{\prime} \in[k, k+N+m-1]$. In addition, for subsystems in $l_{1}^{c_{3}}$, we take $\sum_{l_{1}^{c_{3}}}\left\|e_{k+k_{d_{m}}}^{j}\right\|_{Q^{j}}^{2}>\sum_{l_{1}^{c_{3}}} \lambda_{j}$, according to (11). In case of $m$ packets being dropped, $\tilde{\mathrm{P}}_{l_{2}^{c_{3}}}$ can still be made arbitrarily close to zero, but over a larger horizon $[k, k+N+m]$. We have for the $N+m$-step drift

$$
\tilde{\mathrm{P}}_{l_{2}^{c_{3}}} \Delta V\left(e_{k}^{l_{2}^{c_{3}}}, N+m\right)=\tilde{\mathrm{P}}_{l_{2}^{c_{3}}}\left(\left\|A_{j}^{N+m}\right\|_{2}^{2}-1\right) V\left(e_{k}^{l_{2}^{c_{3}}}\right)+\tilde{\xi}_{l_{2}^{c_{3}}}^{b^{+}},
$$

where $\tilde{\xi}_{l_{2}^{c_{3}}}^{b^{+}}=\tilde{\mathrm{P}}_{l_{2}^{c_{3}}} \sum_{l_{2}^{c_{3}}} \mathrm{E}\left[\left\|\sum_{r=1}^{N+m} A_{j}^{N-r} w_{k+r-1}^{j}\right\|_{Q^{j}}^{2}\right]$. Define $\tilde{f}_{l_{2}^{c_{3}}}\left(e_{k}^{l_{2}^{c_{3}}}\right)=\tilde{\epsilon}_{l_{2}^{c_{3}}} V\left(e_{k}^{l_{2}^{c_{3}}}\right)-\tilde{\xi}_{l_{2}^{c_{3}}}^{b^{+}}$, with $\tilde{\epsilon}_{l_{2}^{c_{3}}} \in(0,1]$. We can find a small set $\tilde{\mathcal{D}}_{l_{2}^{c_{3}}}$ such that $\tilde{f}_{l_{2}^{c_{3}}} \geq 1$, and $\Delta V\left(e_{k}^{l_{2}^{c_{3}}}, N+m\right) \leq$ $-\tilde{f}_{l_{2}}$. Hence, the $f$-norm ergodic theorem holds and the ergodicity of the Markov chain (7) is preserved for an interval of length $N+m$ in case of $m$ packet dropouts. Recall that, cases $c_{1}, c_{2}, l_{1}^{c_{3}}$ for which the ergodicity is shown over an interval of length $N$, remain ergodic over larger intervals. Thus

$$
\begin{aligned}
& \Delta V\left(e_{k}, N+m\right) \leq \Delta V\left(e_{k}^{c_{1}}, N+m\right)+\Delta V\left(e_{k}^{c_{2}}, N+m\right) \\
& \quad+\Delta V\left(e_{k}^{l_{1}^{c_{3}}}, N+m\right)+\tilde{\mathrm{P}}_{l_{2}^{c_{3}} \Delta V\left(e_{k}^{l_{2}^{c_{3}}}, N+m\right) \leq-\tilde{f}\left(e_{k}\right)}
\end{aligned}
$$

This confirms the Theorem 1 holds for the overall drift (19), and proves that the Markov chain (7) is $f$-ergodic.

\section{NUMERICAL RESULTS}

To compare the performance of our bi-character medium access design with the pure stochastic policy proposed in [10], we consider an NCS comprised of two heterogeneous classes of subsystems with equal number of plants belonging 


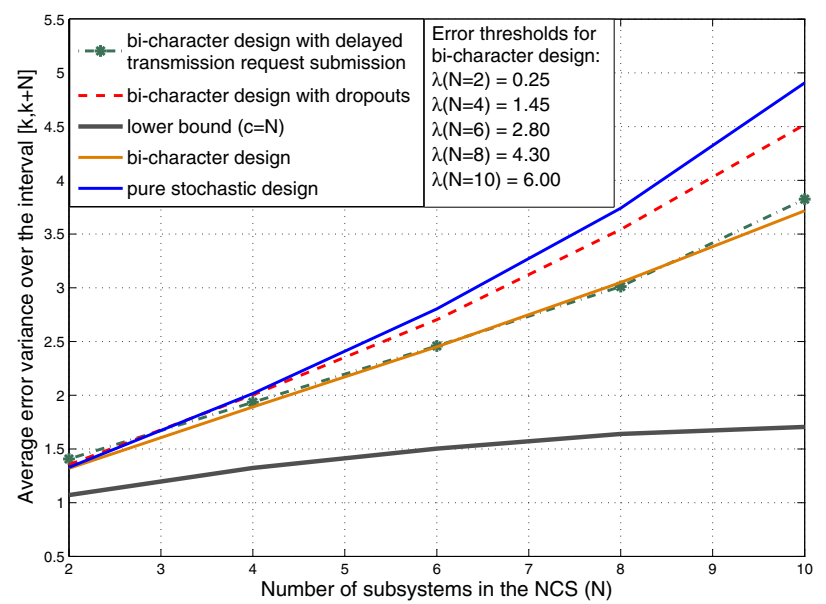

Fig. 2. Comparison of the average mean of error variance vs. the number of control loops for different scheduling policies.

to each class. The first class includes control systems with homogeneous unstable plants while the second class contains loops with homogeneous stable plants. The system parameters are $A_{1}=1.25, B_{1}=1$ and $A_{2}=0.75, B_{2}=1, Q_{i}=I$ and $c=1$. The initial condition is $x_{0}^{1}=x_{0}^{2}=0$ and the noise is given by $w_{k}^{i} \sim \mathcal{N}(0,1)$. To stabilize the loops, we choose a deadbeat control law $L_{i}=A_{i}$ and a model-based observer given by (3).

Figure 2 demonstrates the simulation results providing the comparison between different strategies by considering different number of subsystems $N \in\{2,4,6,8,10\}$. Note that for $N>2$, we have more unstable systems than the available transmission slots per time-step $(c=1)$. The averages are calculated via Monte Carlo simulations over a horizon of $5 \times 10^{5}$. The lower bound is determined by relaxing the constraint in (6) following ideas from [3]. For the sake of simplicity, we calculate the mean variance by considering equal $\lambda$ 's for NCSs with different $N$, as shown in Figure 2. The increase of $\lambda$ as the number of loops increases follows from the fixed channel capacity. The results indicate that employing the bi-character design leads to smaller average error variance compared to the stochastic protocol [10]. The mean variances are also calculated with the same thresholds $\lambda$ considering one packet dropout for an unstable control loop. The variance expectedly increases compared to the case ignoring packet dropouts. We also demonstrate simulation results considering a randomly chosen delay time $d_{k}^{i} \in$ $[0, N-1]$ for two subsystems; one stable and one unstable. As it can be seen, the error variance is slightly different compared to the ideal case, i.e. $d_{k}^{i}=0$. This observation is expected since a delayed transmission request might lead to a higher chance of channel access for a specific system (as the corresponding error might decrease over time). Moreover, even if a transmission request with the highest priority is received undelayed, a transmission is not guaranteed. Thus the average variances remain close even in case of receiving the request with delay.

\section{CONCLUSIONS}

In this paper, we study stability of heterogeneous multiloop NCSs over shared communication channels in terms of ergodicity of the error Markov chain, employing an errordependent scheduling scheme which exhibits both deterministic and probabilistic properties. We demonstrate robustness of our proposed approach against the imperfections such as packet dropouts and delayed transmission requests from local entities. Numerical results illustrate the boundedness of the error variance and display robustness as well as scalability as the number of subsystems increases.

\section{REFERENCES}

[1] P. Tabuada, "Event-triggered real-time scheduling of stabilizing control tasks," Automatic Control, IEEE Transactions on, vol. 52, no. 9, pp. $1680-1685,2007$.

[2] A. Molin and S. Hirche, "On the optimality of certainty equivalence for event-triggered control systems," Automatic Control, IEEE Transactions on, vol. 58, no. 2, pp. $470-474,2013$.

[3] A. Molin and S. Hirche, "A bi-level approach for the design of eventtriggered control systems over a shared network," Discrete Event Dynamic Systems, vol. 24, no. 2, pp. 153-171, 2014.

[4] W. Heemels, J. H. Sandee, and P. Van Den Bosch, "Analysis of eventdriven controllers for linear systems," International Journal of Control, vol. 81 , no. 4, pp. 571-590, 2008.

[5] D. Nesic and A. Teel, "Input-output stability properties of networked control systems," Automatic Control, IEEE Transactions on, vol. 49, no. 10, pp. 1650-1667, 2004.

[6] G. C. Walsh, H. Ye, and L. G. Bushnell, "Stability analysis of networked control systems," Control Systems Technology, IEEE Transactions on, vol. 10, no. 3, pp. 438-446, 2002.

[7] C. Ramesh, H. Sandberg, and K. Johansson, "Stability analysis of multiple state-based schedulers with csma," in Decision and Control (CDC), 2012 IEEE 51st Conference on, pp. 7205-7211, Dec 2012.

[8] M. Donkers, W. Heemels, D. Bernardini, A. Bemporad, and V. Shneer, "Stability analysis of stochastic networked control systems," Automatica, vol. 48, no. 5, pp. 917-925, 2012.

[9] M. Tabbara and D. Nesic, "Input-output stability of networked control systems with stochastic protocols and channels," Automatic Control, IEEE Transactions on, vol. 53, no. 5, pp. 1160-1175, 2008.

[10] M. Mamduhi, A. Molin, and S. Hirche, "Event-based scheduling of multi-loop stochastic systems over shared communication channels," in 21 st International Symposium on Mathematical Theory of Networks and Systems (MTNS), pp. 266-273, Jul 2014.

[11] S.-L. Dai, H. Lin, and S. Ge, "Scheduling-and-control codesign for a collection of networked control systems with uncertain delays," Control Sys. Tech., IEEE Transactions on, vol. 18, no. 1, pp. 66-78, 2010.

[12] M. Ljesnjanin, D. Quevedo, and D. Nesic, "Robustness of networked control systems with multiple actuator-links and bounded packet dropouts," in Decision and Control (CDC), 2013 IEEE 52nd Annual Conference on, pp. 5963-5968, Dec 2013.

[13] M. Branicky, S. Phillips, and W. Zhang, "Stability of networked control systems: explicit analysis of delay," in American Control Conference, vol. 4, pp. 2352-2357 vol.4, 2000.

[14] S. Kar, B. Sinopoli, and J. Moura, "Kalman filtering with intermittent observations: Weak convergence to a stationary distribution," Automatic Control, IEEE Transactions on, vol. 57, pp. 405-420, Feb 2012.

[15] H. Lin, G. Zhai, and P. Antsaklis, "Robust stability and disturbance attenuation analysis of a class of networked control systems," in Decision and Control, 2003. Proceedings. 42nd IEEE Conference on, vol. 2, pp. 1182-1187, 2003.

[16] M. Mamduhi, A. Molin, and S. Hirche, "Event-triggered scheduling for stochastic multi-loop networked control systems with packet dropouts," in Decision and Control (CDC), 2014 53rd IEEE Annual Conference on, pp. 2776-2782, Dec 2014.

[17] S. Meyn and R. Tweedie, Markov chains and stochastic stability. Springer London, 1996.

[18] S. Meyn and R. Tweedie, "State-dependent criteria for convergence of markov chains," The Annals of Applied Probability, pp. 149-168, 1994. 